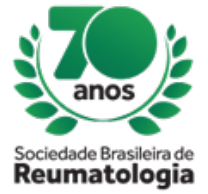

\title{
PROFILE OF PATIENTS USING ANABOLIC THERAPY WITH TERIPARATIDE
}

Elisa Pereira Vicentini (HSPE, São Paulo, SP, Brasil), Estevão da Costa Santana (HSPE, São Paulo, SP, Brasil), Luíza Trincado (HSPE, São Paulo, SP, Brasil), Sandra Echazú Roman (HSPE, São Paulo, SP, Brasil), Ana Paula Dauricio de Mattos (HSPE, São Paulo, SP, Brasil), André Ozela Augusto (HSPE, São Paulo, SP, Brasil), Elaine de Azevedo (HSPE, São Paulo, SP, Brasil), Rina Dalva Neubarth Giorgi (HSPE, São Paulo, SP, Brasil)

\section{BACKGROUND}

Osteoporosis (OP) is a skeletal disorder whose main characteristic is the compromise of strength and bone resistance, predisposing to an increased risk of fracture. Among the therapeutical options available, Teriparatide (TPD) is highlighted. It has been used since 2002 as an anabolic agent for individuals with severe osteoporosis who are at high risk for fractures. In this work, we will discuss the profile of patients with osteoporosis selected for anabolic therapy with TPD followed in a referenced service.

\section{MATERIALS AND METHODS}

A review of 48 medical records, belonging to patients with TPD, with a mean age of 71.47 years, with a diagnosis of osteoporosis was carried out, and the main characteristics, such as time of illness, presence of fragility fractures and the main sites of fracture, among other information.

\section{RESULTS}

Regarding gender and ethnicity, of the patients analyzed, 46 were female and 2 were male. The mean follow-up time for osteoporosis is 13.2 years. In the distribution by age group, there is a higher prevalence of indication for treatment among patients between $60-75$ years, corresponding to $43.75 \%(n=21)$. This is followed by $41.66 \%(n=20)$ of patients over 75 years of age and $14.58 \%(n=7)$ of young patients under 60 years of age. Among the 7 younger patients, $42.85 \%(n=3)$ had osteoporosis secondary to glucocorticoid use, $42.85 \%(n=3)$ presented therapeutic failure with the use of Bisphosphonates and $14.28 \%(n=1))$ had a diagnosis of osteogenesis imperfecta. Of all 48 patients, $85.42 \%(n=41)$ had atraumatic fractures. Among the sites affected: $41.67 \%(n=20)$ lumbar and thoracic spine, $31.25 \%(n=$ 15) femur, $25 \%(n=12), 10.41 \%(n=5) 2.08 \%(n=1)$ clavicle and $16.66 \%(n=8)$ at other sites. Twelve patients, that is, $25 \%$ presented fractures at multiple sites.

\section{CONCLUSION}

It is noteworthy that patients with indication for anabolic therapy are elderly, female and severe osteoporosis. Most of these patients already had atraumatic fractures and the most frequent site is the spine. This place predisposes to serious injuries, which may compromise patient mobility and functionality. Within the group of young patients, the secondary causes of osteoporosis are highlighted, mainly the chronic use of glucocorticoids 